\title{
AM-FM Picture Carrier Beat Type Noise Filters
}

\author{
Sahithi Peddireddy, Nick Mould, and Joseph P. Havlicek \\ University of Oklahoma \\ School of Electrical and Computer Engineering \\ Norman, OK, USA \\ sahithi@ou.edu,nick.mould@gmail.com,joebob@ou.edu
}

\begin{abstract}
For the first time, modulation domain filters are proposed for removing beat type noises in video corrupted by unwanted external signals during the transmission process. We demonstrate the effectiveness of our proposed algorithm on picture carrier beat type noise observed in a real-world situation involving the transmission of critical video signals obtained from a traffic monitoring system. We compare and contrast our results with a traditional linear time invariant (LTI) notch filter. The technique can be generalized for removal of a variety of fixed pattern noises characteristic of many infrared and other non-visible wavelength electro-optical detectors. In addition, we provide a theoretical comparison of modulation and frequency domain image representation strategies in the context of digital image filtering.
\end{abstract}

Index Terms-AM-FM image models, AM-FM image filters, modulation domain signal processing, multicomponent models

\section{INTRODUCTION}

Fourier image representations characterize image structure in terms of complex sinusoidal functions with constant amplitude and frequency components; thus they do not provide intuitive descriptions for nonstationary visual textures. Nonstationary textural image features are better characterized in terms of instantaneously varying quantities such as instantaneous amplitude and instantaneous frequency that are more closely related to human visual perception and of great research interest [1]-[14]. In contrast to Fourier analysis, AM-FM image models represent the image directly in terms of instantaneous amplitude and frequency functions expressed as nonstationary complex sinusoidal signals.

In the past, the representation of images in terms of AMFM functions has resulted in valuable knowledge of the spatially localized amplitudes and frequencies of textural image features that have been used with great success in a wide variety of computer vision and analysis applications, including image segmentation [2], [3], [6], [7], [13]-[16], content based image retrieval [17], regeneration of occluded and damaged textures [18], human speech analysis [4], [19], fingerprint analysis and classification [5], [20], target tracking [11], [12], estimation of 3-D shape from texture [14], [21], infrared target tracking [12], and visible target tracking [22]. Recently, AMFM models have been used to develop modulation domain image filtering algorithms [10], allowing for filtering techniques and results which are not possible using the classical Fourier representation.

Computing the AM-FM functions of an image is an ill posed problem. There are significant difficulties and several different approaches have been proposed over the past few years. For any real-valued image, there exist an infinite number of AM-FM representations in terms of a single pair of modulating functions. Moreover, multicomponent models are generally more desirable and the decomposition of an image into components is also non-unique. To overcome this problem we compute a complex extension of the image using the two dimensional Hilbert transform [2]. Such a complex image yields unique modulation functions. It is of practical interest to produce smoothly varying and thus visually meaningful AMFM functions. But for real valued images this is not possible using a single AM-FM function. It is necessary to divide the image into components so that smoothly varying AMFM functions can be obtained. This is called multi-component AM-FM image modeling. The computation of FM functions for discrete cases is a difficult one as the gradient is not welldefined for discrete images. Recently, an algorithm was developed which gives exact modulating functions in the discrete case [8], and modulation domain filtering is now possible in the context of a perfect reconstruction filterbank. Modulation domain filtering can obtain results that are impossible to obtain with traditional LTI filters. The concept of modulation domain filtering has been recently developed and there are many interesting possibilities that remain largely unexplored.

This paper presents one such unexplored application area: improving the quality of cable television signals. Television signals transmitted at the head end of a TV cable system should be equal to the signals received at the receiving end. Practically, this is not the case. The signal at the receiving end is generally degraded due to the intrusion of unwanted noise signals during transmission. Intermodulation distortion of carrier signals causes picture carrier beat noise. Picture carrier beat noise produces diagonal moving bars in the video signal. This paper uses the concept of AM-FM image models to process the instantaneous amplitude and frequency functions and reduce picture carrier beat noise. This is a new and important potential application of modulation domain filtering.

\section{AM-FM IMAGE MODELS}

For a real valued signal, $s: \mathbb{R}^{n} \rightarrow \mathbb{R}$, where $\mathrm{x} \in \mathbb{R}^{n}$, the AM-FM signal model is given by [13], [23]

$$
s(\mathbf{x})=a(\mathbf{x}) \cos [\varphi(\mathbf{x})] .
$$


For a complex valued signal $z: \mathbb{R}^{n} \rightarrow \mathbb{C}$, the AM-FM signal model is given by [23]

$$
z(\mathbf{x})=a(\mathbf{x}) e^{j \varphi(\mathbf{x})},
$$

where $a: \mathbb{R}^{n} \rightarrow[0, \infty)$ is the amplitude modulation function and $\varphi: \mathbb{R}^{n} \rightarrow \mathbb{R}$ is the phase modulation of the signal. The frequency modulation function $\nabla \varphi: \mathbb{R}^{n} \rightarrow \mathbb{R}^{n}$ in both cases is the gradient of the phase modulation.

AM-FM functions represent the signal amplitude and frequency at a fine spatial scale. For example, consider a onedimensional speech signal with respect to time. The modulation functions embody the jointly localized structure of the signal, giving frequency with respect to time. This is unlike the Fourier transform which interprets the signal in terms of a collection of constant amplitude and linear phase terms. The AM-FM functions are often called instantaneous amplitude, phase and frequency [3], [8].

\section{A. The Analytic Image}

Gabor introduced an important complex extension for real one-dimensional signals in [24]. For a real signal $s(t)$ Gabor's analytic signal is given by

$$
z(t)=s(t)+j q(t)
$$

where $q(t)$ is defined by

$$
q(t)=\mathcal{H}[s(t)]=s(t) * \frac{1}{\pi t}=\frac{1}{\pi} \int_{\mathbb{R}} \frac{s(\tau)}{t-\tau} d \tau,
$$

and $\mathcal{H}[\cdot]$ is the $1 \mathrm{D}$ Hilbert transform. The integral in (4) is evaluated as a Cauchy principal value. The signal $z(t)$ is complex and is referred to as the analytic signal. The analytic signal obtained in this process has a unique set of modulation functions. The analytic signal obtained using Hilbert transform admits some attractive properties. The spectral redundancy in the real signal is eliminated [25], i.e., the analytic spectrum $Z(\Omega)$ is zero for all negative frequencies, having support only for the positive frequencies. Thus, it represents the removal of the redundant frequency content in real-valued signals. Further properties were also shown by Vakman [26]. It is important for us to be able to extend the 1D Hilbert transform to 2D so that it can be used for images. The $2 \mathrm{D}$ complex extension of a real valued image using the partial Hilbert transform is referred to as the analytic image and is given by [25],

$$
z(\mathbf{x})=s(\mathbf{x})+j q(\mathbf{x}),
$$

where $q(\mathbf{x})$ is the partial Hilbert transform.

The partial Hilbert transform can be applied to any multidimensional signal $s(\mathbf{x}): \mathbb{R}^{n} \rightarrow \mathbb{R}$ where $\mathbf{x}=\left[\begin{array}{llll}x_{1} & x_{2} & \ldots & x_{n}\end{array}\right]^{T}$, but is defined as having a single direction of action by a unit vector in the $x_{i}$ direction. It has a standard definition [27]-[29] which is given by

$$
\mathcal{H}[s(\mathbf{x})]=\frac{1}{\pi} \int_{\mathbb{R}} s\left(\mathbf{x}-\xi \mathbf{e}_{i}\right) \frac{d \xi}{\xi}=s(\mathbf{x}) * \frac{1}{\pi \mathbf{x}^{T} \mathbf{e}_{i}} \prod_{k \neq i} \delta\left(\mathbf{x}^{T} \mathbf{e}_{k}\right),
$$

where $\mathbf{e}_{i}$ is the unit vector in the $x_{i}$ direction and the integral in (6) is interpreted as a Cauchy principal value. The analytic image in (6) carries many of the same properties as the 1D analytic signal. In both the cases, reduction of spectral redundancy is achieved. The process of computing the discrete analytic image is analogous to the continuous analytic signal. The discrete analytic image is of interest here as we are using a computer to do the computations and is given in [3], [25]. After the analytic image is generated it is possible to obtain unique amplitude and frequency modulation functions; this is called demodulation.

\section{B. Demodulation}

To decompose the analytic image into locally coherent components, the steerable pyramid filterbank is used [10]. The steerable pyramid filterbank is implemented as in [30], [31]. Here the steerable pyramid decomposition is implemented at five levels and at eight orientations resulting in 40 locally coherent image components. For computational purposes, each channel response in the steerable pyramid is computed in the frequency domain, by pointwise multiplication of DFTS. With this approach, generation of the multicomponent analytic image can be folded into the filterbank without additional overhead [2].

In the demodulation step, the amplitude and frequency modulating functions $a_{k}(\mathbf{x})$ and $\nabla \varphi_{k}(\mathbf{x})$ are calculated from the $k$ component image decomposition. In the continuous case the modulating functions can be directly computed from the complex image components $s_{k}$ according to

$$
a_{k}(\mathbf{x})=\left|s_{k}(\mathbf{x})+j q_{k}(\mathbf{x})\right|
$$

and

$$
\nabla \varphi_{k}(\mathbf{x})=\frac{\nabla q_{k}(\mathbf{x}) s_{k}(\mathbf{x})-q_{k}(\mathbf{x}) \nabla s_{k}(\mathbf{x})}{a_{k}(\mathbf{x})} .
$$

In the discrete case, computation of the amplitude modulation function $a_{k}(\mathbf{x})$ is the same as the continuous case [8] but the frequency modulation cannot be calculated by (8) because the gradient is undefined. Instead, we compute the phase modulation from the solution given in [8]. The gradient image for the discrete case is calculated using tensor product spline models proposed by Unser et al., [32]-[34] to interpolate a discrete image and to analytically define its gradient $\nabla s_{k}(\mathbf{x})+j \nabla q_{k}(\mathbf{x})$. But the phase modulations are ambiguous at pixels where the amplitude function is zero. For such pixels, interpolation is performed by averaging the phase at the neighboring pixels. The obtained phase functions are consistent with human visual perception and do not suffer from phase discontinuities in $(-\pi, \pi]$ when inverse trigonometric functions are combined with the spline-based algorithm proposed in [8].

We compute the wrapped phase according to [8]

$$
W\{\cdot\}=\arctan \left[\frac{q_{k}(\mathbf{x})}{s_{k}(\mathbf{x})}\right],
$$

where $W\{\cdot\}$ is the wrapping operator that maps each phase value to its modulo- $2 \pi$ congruent value in $(-\pi, \pi]$. The desired 
unwrapped phase can be expressed as [8]

$$
\varphi_{k}(x)=W\left\{\varphi_{k}(\mathbf{x})\right\}+2 \pi b_{k}(\mathbf{x}) \approx \gamma \psi_{k}(\mathbf{x}),
$$

where $W\left\{\varphi_{k}(\mathbf{x})\right\}$ is the wrapped phase modulation, the branch function $b_{k}(\mathbf{x})$ is defined as in (11), $\gamma \psi_{k}(\mathbf{x})$ is the scaled phase function, $\gamma$ is a phase constant and $\psi_{k}(\mathbf{x})$ is the estimated phase modulation computed from the spline based demodulation. The branch function $b(\mathbf{x})$ in (10) is given by

$$
b_{k}(\mathbf{x})=\operatorname{round}\left(\frac{\gamma \psi_{k}(\mathbf{x})-W\left\{\varphi_{k}(\mathbf{x})\right\}}{2 \pi}\right) .
$$

Here, $\gamma$ is equal to 300 .

The gradient of the phase modulation gives the frequency modulation function. The gradient is computed analytically in the horizontal and vertical directions defined as $\nabla \varphi_{k}(\mathbf{x})$. The obtained frequency modulation functions are scaled by the value of the phase constant $\gamma$. Finally, the obtained phase modulation functions are scaled by the value of $\gamma$.

\section{EXPERIMENTAL RESULTS}

\section{A. Data Acquisition}

NTSC Video signals were captured from a live video stream in a highway surveillance system. The video signal was preprocessed to convert from the RGB to grayscale feature space. Lastly, the acquired video was corrupted by combining the signal with a constant carrier waveform of the same radio frequency, resulting in picture carrier beat distortions manifested in the video as scrolling diagonal bars.

\section{B. Modulation domain filtering}

A modulation domain picture carrier beat type noise removal filter based on the component amplitude functions $a_{k}(\mathbf{x})$, frequency magnitudes $r_{k}(\mathbf{x})=\left|\nabla \varphi_{k}(\mathbf{x})\right|$ and frequency directions $\theta_{k}(\mathbf{x})=\arg \nabla \varphi_{k}(\mathbf{x})$ is proposed. The filter is specified by

$$
\hat{a}_{k}(\mathbf{x})=\left\{\begin{aligned}
a_{k}(\mathbf{x}) \cdot \frac{0.9-r_{k}(\mathbf{x})}{0.05}, & 0.85<r_{k}(\mathbf{x})<0.9 \\
0, & 0.9 \leq r_{k}(\mathbf{x}) \leq 1.8 \\
a_{k}(\mathbf{x}) \cdot \frac{r_{k}(\mathbf{x})-1.8}{0.05}, & 1.8 \leq r_{k}(\mathbf{x}) \leq 1.85 \\
0, & 0.6 \leq r_{k}(\mathbf{x}) \leq 0.825 \\
& -\pi / 2 \leq \theta_{k}(\mathbf{x})<0 \\
a_{k}(\mathbf{x}), & \text { otherwise }
\end{aligned}\right.
$$

Fig. 1 shows a comparison the results obtained by application of the modulation domain filter proposed in (12) and a comparable LTI filter designed by manual estimation of the picture beat noise statistics. Fig.1(a, d and g) depict three frames of a real-world image sequence obtained from a stationary traffic camera at I35 and 27th street in Oklahoma City, Oklahoma that have been corrupted by picture carrier beat noise. Fig. 1(b, e and h) are the LTI filter results and, (c, $\mathrm{f}$ and $\mathrm{i}$ ) are the modulation domain filter results. In both cases, the picture carrier beat noise has been significantly attenuated, however, the results obtained with the LTI filter contain a significant number of unwanted ringing artifacts With respect to the modulation domain filtering results, the only significant
TABLE I

QUANTITATIVE REPRESENTATION OF THE RESULT FRAMES IN TERMS OF STANDARD ERROR MEASUREMENTS PSNR AND ISNR.

\begin{tabular}{|c|c|c|c|c|c|}
\cline { 2 - 6 } & \multicolumn{3}{c|}{ PSNR } & \multicolumn{2}{c|}{ ISNR } \\
\cline { 2 - 6 } & $I$ & $\hat{I}_{(L T I)}$ & $\hat{I}_{(R, \theta)}$ & $\hat{I}_{(L T I)}$ & $\hat{I}_{(R, \theta)}$ \\
\hline \hline For 1 Frame & 27.0253 & 26.7885 & 29.8198 & -0.2367 & 2.7946 \\
\hline For 20 Frames & 26.9929 & 26.6462 & 28.7921 & -0.3467 & 1.7992 \\
\hline \hline
\end{tabular}

drawback is the smoothing of sharp edges that can be observed in Fig. 1(c, f and i).

In Table I a quantitative comparison of the LTI filter and the modulation domain filter is provided in terms of peak signal to noise ratio (PSNR) and improvement in signal to noise ratio (ISNR). In all cases, the performance of the modulation domain filter are superior to those obtained through LTI filtering.

\section{ACKNOWLEDGMENT}

This work was supported in part by the U.S. Army Research Laboratory and the U.S. Army Research Office under grant W911NF-08-1-0293.

\section{REFERENCES}

[1] J. P. Havlicek, D. S. Harding, and A. C. Bovik, "Reconstruction from the multi-component AM-FM image representation," in Proc. IEEE Int'l. Conf. Image Proc., Washington, DC, Oct. 22-25, 1995, vol. II, pp. 280-283.

[2] J. P. Havlicek, D. S. Harding, and A. C. Bovik, "Multidimensional quasieigenfunction approximations and multicomponent AM-FM models," IEEE Trans. Image Proc., vol. 9, no. 2, pp. 227-242, Feb. 2000.

[3] J. P. Havlicek, "The evolution of modern texture processing," Elektrik, Turkish Journal of Electrical Engineering and Computer Sciences, vol. 5, no. 1, pp. 1-28, 1997.

[4] P. Maragos, J. F. Kaiser, and T. F. Quatieri, "Energy separation in signal modulations with applications to speech analysis," IEEE Trans. Signal Proc., vol. 41, no. 10, pp. 3024-3051, Oct. 1993.

[5] N. Kitiyanan and J. P. Havlicek, "Modulation domain reference point detection for fingerprint recognition," in Proc. IEEE Southwest Symp. Image Anal., Interp., Lake Tahoe, NV, Mar. 28-30, 2004, pp. 147-151.

[6] T. Tangsukson and J. P. Havlicek, "Modulation domain image segmentation," in Proc. IEEE Southwest Symp. Image Anal., Interp., Austin, TX, Apr. 2-4, 2000, pp. 45-50.

[7] T. Tangsukson and J. P. Havlicek, "AM-FM image segmentation," in Proc. IEEE Int'l. Conf. Image Proc., Vancouver, BC, Canada, Sep. 1013, 2000, vol. II, pp. 104-107.

[8] R. A. Sivley and J. P. Havlicek, "Perfect reconstruction AM-FM image models," in Proc. IEEE Int'l. Conf. Image Proc., Atlanta, GA, Oct. 8-11, 2006, pp. 2125-2128.

[9] R. A. Sivley and J. P. Havlicek, "A spline-based framework for perfect reconstruction AM-FM models," in Proc. IEEE Southwest Symp. Image Anal., Interp., Denver, CO, Mar. 26-28, 2006, pp. 198-202.

[10] C. T. Nguyen and J. P. Havlicek, "AM-FM image filters," in Proc. IEEE Int'l. Conf. Image Proc., San Diego, CA, Oct. 12-15, 2008, pp. 789-792.

[11] C. T. Nguyen and J. P. Havlicek, "Modulation domain features for discriminating infrared targets and backgrounds," in Proc. IEEE Int'l. Conf. Image Proc., Atlanta, GA, Oct. 8-11, 2006, pp. 3245-3248.

[12] C. T. Nguyen, J. P. Havlicek, and M. Yeary, "Modulation domain template tracking," in Proc. IEEE Int'l. Conf. Image Proc., San Antonio, TX, June 17-22, 2007, pp. 1-8.

[13] A. C. Bovik, M. Clark, and W. S. Geisler, "Multichannel texture analysis using localized spatial filters," IEEE Trans. Pattern Anal. Machine Intell., vol. 12, no. 1, pp. 55-73, Jan. 1990.

[14] A. C. Bovik, N. Gopal, T. Emmoth, and A. Restrepo, "Localized measurement of emergent image frequencies by Gabor wavelets," IEEE Trans. Info. Theory, vol. 38, no. 2, pp. 691-712, Mar. 1992. 

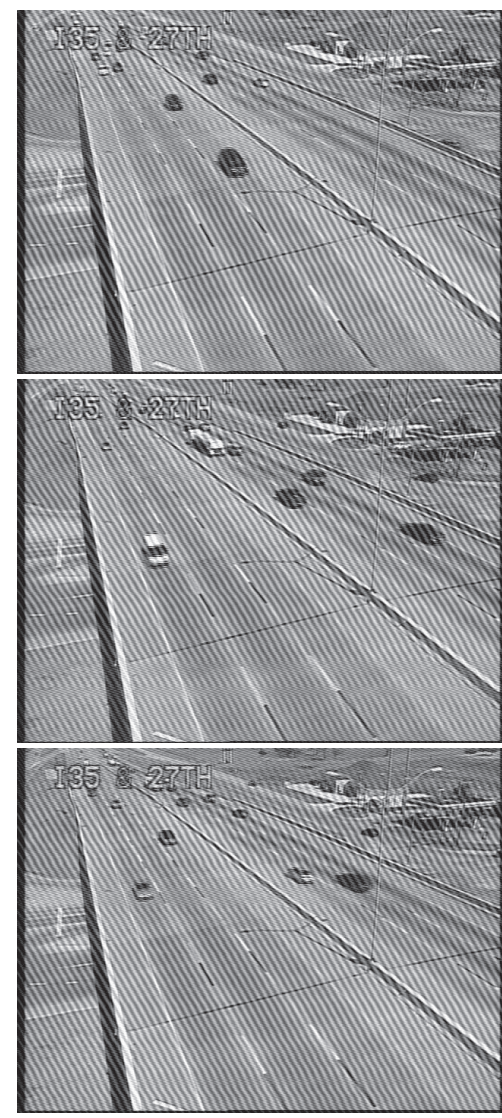

(d)

(a)
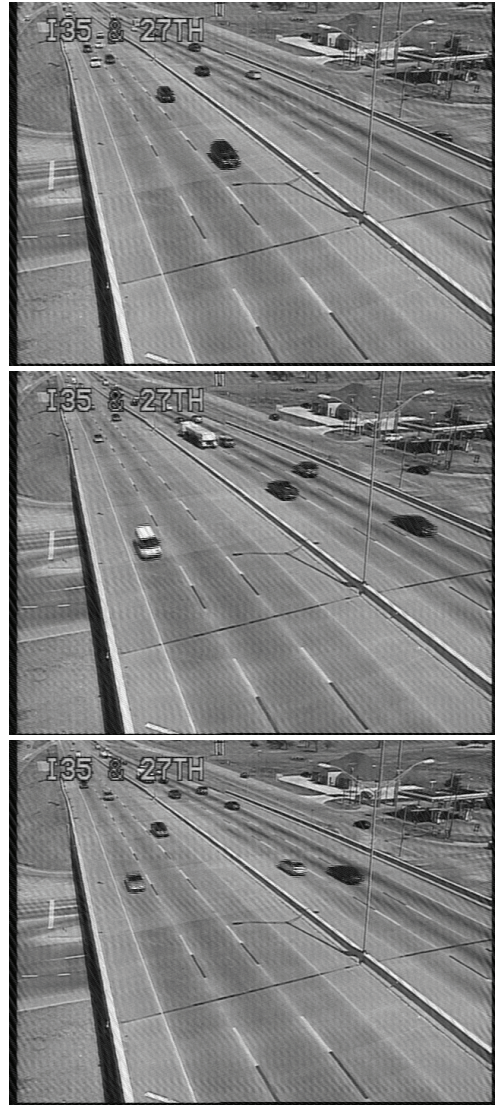

(e)

(b)
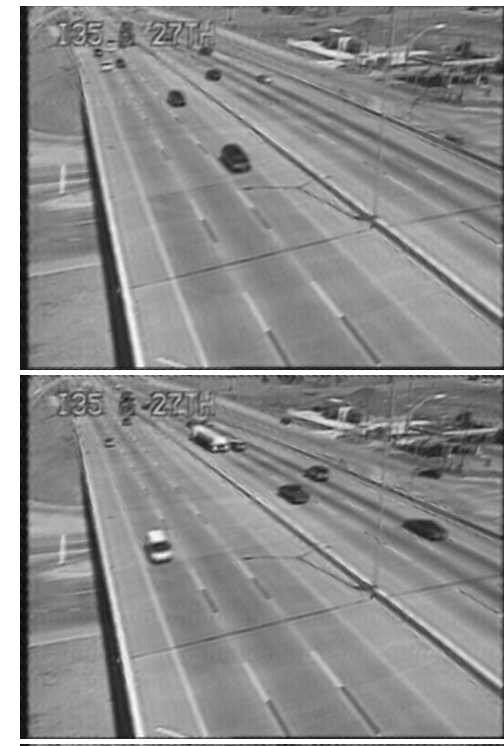

(c)

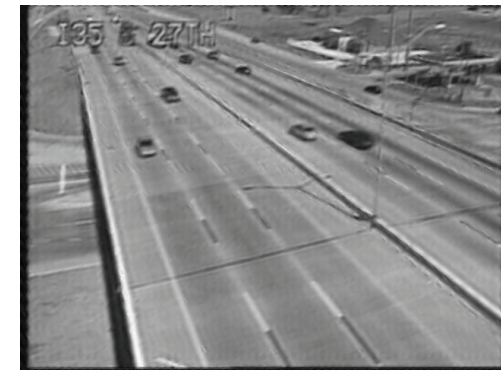

(f)

(h)

Fig. 1. Selected frames depicting images corrupted by picture carrier beat noise (a, d, g), and noise filtering results obtained by LTI filtering (b, e, h) and modulation domain filtering (c, $\mathrm{f}, \mathrm{i})$.

[15] A. C. Bovik, "Analysis of multichannel narrow-band filters for image texture segmentation," IEEE Trans. Signal Proc., vol. 39, no. 9, pp. 2025-2043, Sep. 1991.

[16] T. B. Yap, T. Tangsukson, P. C. Tay, N. D. Mamuya, and J. P. Havlicek, "Unsupervised texture segmentation using dominant image modulations," in Proc. 34th IEEE Asilomar Conf. Signals, Syst. Comput., Pacific Grove, CA, Oct. 29-Nov. 1, 2000, vol. II, pp. 911915.

[17] J. P. Havlicek, J. Tang, S. T. Acton, R. Antonucci, and F. N. Ouandji, "Modulation domain texture retrieval for CBIR in digital libraries," in Proc. 35th IEEE Asilomar Conf. Signals, Syst., Comput., Pacific Grove, CA, Nov. 9-12, 2003, pp. 1580-1584.

[18] J. P. Havlicek, P. C. Tay, and A. C. Bovik, "AM-FM image models: Fundamental techniques and emerging trends," in Handbook of Image and Video Processing, A. C. Bovik, Ed., pp. 377-395. Elsevier Academic Press, Burlington, MA, 2nd edition, 2005.

[19] T. Ezzat, J. Bouvrie, and T. Poggio, "AM-FM demodulation of spectrograms using localized 2D max-Gabor analysis," in Proc. IEEE Int'l Conf. Acoust., Speech, Signal Process., Apr. 15-20, 2007 vol. IV, pp. 1061-1064.

[20] M. S. Pattichis, G. Panayi, A. C. Bovik, and S. -P. Hsu, "Fingerprint classification using an AM-FM model," IEEE Trans. Image Proc., vol. 10, no. 6, pp. 951-954, Jun. 2001.

[21] B. J. Super and A. C. Bovik, "Shape from texture using local spectral moments," IEEE Trans. Pattern Anal. Machine Intell., vol. 17, no. 4, pp. 333-343, Apr. 1995.

[22] R. S. Prakash and R. Aravind, "Modulation-doman particle filter for template tracking," in Proc. Int'l Conf. Pattern Recog., Tampa, FL, Dec 8-11, 2008, pp. 1-4.

[23] J. P. Havlicek, A. C. Bovik, and P. Maragos, "Modulation models for image processing and wavelet-based image demodulation," in Proc. 26th
IEEE Asilomar Conf. Signals, Syst., Comput., Pacific Grove, CA, Oct. 26-28, 1992, pp. 805-810.

[24] D. Gabor, "Theory of communication," J. Inst. Elect. Eng. London, vol. 93, no. III, pp. 429-457, 1946.

[25] J. P. Havlicek, J. W. Havlicek, and A. C. Bovik, "The analytic image," in Proc. IEEE Int'l. Conf. Image Proc., Santa Barbara, CA, Oct. 26-29, 1997.

[26] D. Vakman, "On the analytic signal, the Teager-Kaiser energy algorithm, and other methods for defining amplitude and frequency," IEEE Trans. Signal Proc., vol. 44, no. 4, pp. 791-797, Apr. 1996.

[27] A. P. Calderón and A. Zygmund, "On singular integrals," Am. J. Math., vol. 78, no. 2, pp. 289-309, Apr. 1956.

[28] E. M. Stein, Singular Integrals and Differentiability Properties of Functions, Princeton University Press, Princeton, NJ, 1970.

[29] E. M. Stein and G. Weiss, Introduction to Fourier Analysis on Euclidean Spaces, Princeton University Press, Princeton, NJ, 1971.

[30] E. P. Simoncelli and W. T. Freeman, "The steerable pyramid: a flexible architecture for multi-scale derivative computation," in Proc. IEEE Int'l. Conf. Image Proc., Washington, DC., Oct. 23-26, 1995, pp. 444-447.

[31] E. P. Simoncelli, W. T. Freeman, E. H. Adelson, and D. J. Heeger, "Shiftable multi-scale transform," IEEE Trans. Info. Theory, vol. 38, no. 2, pp. 587-607, March. 1992.

[32] M. Unser, A. Aldroubi, and M. Eden, "Fast B-spline transforms for continuous image representation and interpolation," IEEE Trans. Pattern Anal. Machine Intell., vol. 13, no. 3, pp. 277-285, Mar. 1991.

[33] M. Unser, A. Aldroubi, and M. Eden, "B-spline signal processing: Part I-theory," IEEE Trans. Signal Proc., vol. 41, no. 2, pp. 821-833, Feb. 1993.

[34] M. Unser, A. Aldroubi, and M. Eden, "B-spline signal processing: Part II-efficient design and applications," IEEE Trans. Signal Proc., vol. 41, no. 2, pp. 834-848, Feb. 1993. 